\title{
Sampling Frequency and Digital Noise Filtering for Transient Kinetic Study in CSTR via Temperature Scanning and Composition Modulation Technique
}

\author{
Amaraporn Kaewchada* \\ Department of Agro-Industrial, Food, and Environmental Technology, Faculty of Applied Science, King \\ Mongkut's University of Technology North Bangkok, Bangkok, Thailand \\ Kwanhatai Jiradechkhajorn and Attasak Jaree \\ Department of Chemical Engineering, Faculty of Engineering, Kasetsart University, Bangkok, Thailand \\ * Corresponding author. E-mail: amaraporn.k@sci.kmutnb.ac.th \\ Received: 26 June 2015; Accepted: 13 October 2015; Published online: 28 October 2015 \\ (C) 2015 King Mongkut's University of Technology North Bangkok. All Rights Reserved.
}

\begin{abstract}
Temperature Scanning and Composition Modulation technique (TS-CM) is a technique for estimating reaction parameters under transient conditions in a CSTR reactor. Studies of reaction kinetics performed under transient conditions can lead to savings of time and resources as compared to the conventional steady-state method. This is because a collection of data necessary for extracting the reaction kinetics can be obtained within a single experiment. This research focused on the refinement of TS-CM technique as an efficient tool for reaction kinetics studies. The alkaline hydrolysis of ethyl acetate was used as a model reaction. Different degrees of noise intensity were added to the output signal of $\mathrm{NaOH}$ concentration in order to study the effect of noise $(0.5,1.0,3.0$ and $5.0 \%)$ and sampling period (1,30 and 60 seconds) on the accuracy of reaction kinetics. Results revealed that the sampling period of 1 second yielded only slight errors of kinetic parameters compared to that of without noise. The second part dealt with noise filtering. In order to smooth the $\mathrm{NaOH}$ concentration data, TS-CM technique was applied with three digital filters: a) the first-order filter b) the second-order filter, and c) the Savitzky-Golay filter. Results showed that the best filter was the Savitzky-Golay, which filtered with 81 points of window size and $2^{\text {nd }}$ order of polynomial degree.
\end{abstract}

Keywords: Transient kinetics, Temperature scanning, Composition modulation, Noise filtering

\section{Introduction}

Newly discovered chemical reactions undoubtedly must be investigated to obtain information about stoichiometry and reaction kinetics. This crucial information will be used to optimize the production rate in terms of operating conditions, to design the chemical reactor or to develop the production facility, to study dynamics of such chemically reacting systems for designing controller, to propose the reaction mechanism, to design catalysts, etc. [1]. In chemically reacting systems, the intrinsic reaction kinetics can be obscured by the molecular diffusion. Recent developments on reactor technology have shown that many reactions perform better in a micro-scale reactor compared to the macro-scale reactor [2], [3]. Hence, one must be cautious about the system used to obtain information on reaction kinetics. The kinetic studies can be performed in either batch or flow reactors by following the extent of reaction with the change of operating conditions such as residence time, reaction temperature, pressure, or the molar ratio of reactants.

Please cite this article as: A. Kaewchada, K. Jiradechkhajorn, and A. Jaree, "Sampling Frequency and Digital Noise Filtering for Transient Kinetic Study in CSTR via Temperature Scanning and Composition Modulation Technique," KMUTNB Int J Appl Sci Technol, Vol. 8, No. 4, pp. 283-292, 2015, http://dx.doi.org/10.14416/j.ijast.2015.10.002 
By and large, steady-state experiments are performed to collect such data. The nature of steady-state experiment requires a lot of resources. Measurements and analysis of samples must be performed in order to verify the steady-state assumption for the kinetic modelling. This problem is rather amplified if a large set of data is required to increase the accuracy of the kinetic parameters.

Applying transient modeling to the investigation of reaction kinetics can significantly simplify the experimental procedure with reduced demand for time and resources necessary to obtain all data. This way, the laborious procedure of gathering steady-state data can be avoided. There are several categories of transient experiments for kinetic investigation. Temperature scanning (TS) is a technique that forces the continuous change of extent of reaction upon the trajectory of reaction temperature [4]. A collection of data obtained during transient experiment can be used to associate with the profile generated by the transient simulation using the proposed kinetic model. This leads to savings of time and resources required for experimentation as compared to the conventional steady-state ones. Generally, a continuous ramp of reaction temperature is employed for TS experiments [5]-[7].

Another technique of transient experiment for kinetic studies is called composition modulation (CM). By applying cyclical forced concentration to a chemically-reacting system, the pattern of output concentration response can be used to associate with the reaction rate expression. The forcing function can be sinusoidal or square waves. Apart from the empirical form of rate expression, the data collected from $\mathrm{CM}$ experiment have been used to study the reaction mechanism [4]. This technique can also be used to enhance the performance of chemical reactors [8], [9]. Recently, a combination of temperature scanning and composition modulation (TS-CM) was demonstrated as an efficient data gathering technique for extracting information on reaction kinetics [10]. The TS-CM method couples the changes of reaction temperature and concentration while collecting the extent of reaction throughout the experiment. Analysis of data yielded the same reaction orders and very close values of pre-exponential factor and the activation energy compared to that obtained from steady-state kinetic studies. The composition modulation has also been applied with specialized equipments for studies of surface reaction under vacuum [11]. Controlled pulsing of reactant molecules can be arranged in order to monitor the response pattern. This technique is known as the temporal analysis of products (TAP).

Practically, the quality of data collected during transient experiments depends on the instrumentation involved. In general, there is a certain degree of noise embedded in the stream of data due to the accuracy and repeatability of measuring device, electrical interferences, the nature of system or process, etc. [12]. In this contribution, the effect of noise intensity and sampling period on the accuracy of kinetic information extracted via TS-CM technique is investigated. The alkaline hydrolysis of ethyl acetate is used as a model reaction. Three different techniques including an exponential filter or first-order filter, a double exponential filter or second-order filter, and a SavitzkyGolay filter are applied to enhance the performance of TS-CM technique.

\section{Materials and Methods}

\subsection{Experimental procedure}

Experiments for the alkaline hydrolysis of ethyl acetate in the TS-CM CSTR were performed using a method described by Jaree and Nuammaneerat [10]. Figure 1 shows the experimental setup for a kinetic study by TS-CM CSTR. $0.02 \mathrm{M}$ of $\mathrm{NaOH}$ and $0.012 \mathrm{M}$ of ethyl-acetate solutions were stored in their reservoirs. The operation was started by feeding $0.02 \mathrm{M} \mathrm{NaOH}$ solution at a volumetric flow rate $74 \mathrm{~cm}^{3} / \mathrm{min}$ by peristaltic pump (easy-Load II by Master flex) from the solution reservoir into the mixing tank. The mixing tank is a $330 \mathrm{~cm}^{3}$ glass vessel fitted with magnetic stirrer hotplate and a pH-meter. The outflow of $\mathrm{NaOH}$ solution from the mixing tank was transferred into a CSTR. A stream of $0.012 \mathrm{M}$ ethyl-acetate solution was fed from a reservoir at a volumetric flow rate of 67.5 $\mathrm{cm}^{3} / \mathrm{min}$ to the CSTR, which was controlled at $31.2^{\circ} \mathrm{C}$. The CSTR reactor is a $330 \mathrm{~cm}^{3}$ glass vessel fitted with magnetic stirrer hotplates and a conductivity measuring probe (Cond 330i/SET\&TatraCon 325). After the system had reached steady state, $5 \mathrm{~cm}^{3}$ of $0.8 \mathrm{M} \mathrm{NaOH}$ solution was injected to the mixing tank while the temperature in the CSTR reactor was increased from $31.2^{\circ} \mathrm{C}$ to $45^{\circ} \mathrm{C}$ with a ramping rate of $0.02^{\circ} \mathrm{C} / \mathrm{sec}$. Readings from the $\mathrm{pH}$-meter, conductivity meter, 


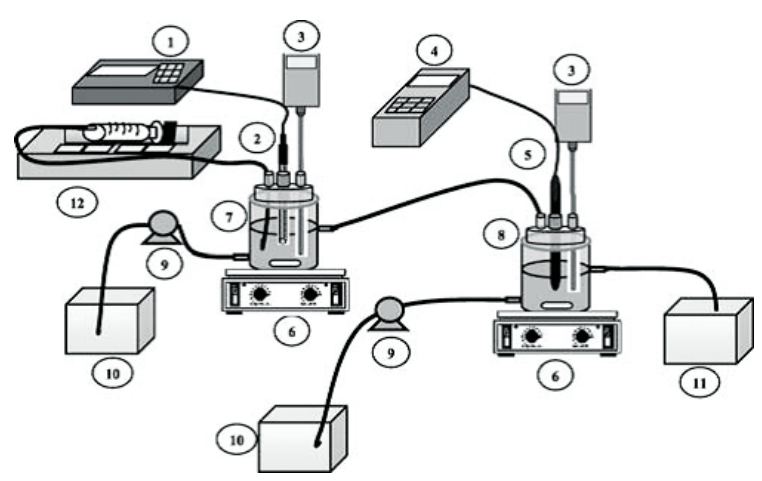

Figure 1: Experimental set-up: (1) $\mathrm{pH}$ meter, (2) $\mathrm{pH}$ measuring probe, (3) Thermometer sensor, (4) conductivity measuring cell, (5) conductivity measuring probe, (6) magnetic stirrer hotplates, (7) mixing tank, (8) CSTR, (9) pump, (10) solution reservoir, (11) sample reservoir, (12) syringe pump.

and thermocouple display were recorded during the experiment. These data will later be used to extract kinetic information.

\subsection{Kinetic and reaction model}

In this part of our work we will illustrate a theoreticalmathematical model of the hydrolysis of ethyl acetate integrated with TS-CM CSTR technique. First, in order to demonstrate our special technique, the common reaction kinetics is explained. Ethyl acetate hydrolyzes in alkaline solution as shown in equation (1).

$$
\begin{aligned}
& \mathrm{NaOH}+\mathrm{CH}_{3} \mathrm{CO}_{2} \mathrm{C}_{2} \mathrm{H}_{5} \rightarrow \mathrm{C}_{2} \mathrm{H}_{5} \mathrm{OH}+\mathrm{CH}_{3} \mathrm{CO}_{2} \mathrm{Na} \\
& \text { (A) } \\
& \text { (B) }
\end{aligned}
$$

From the stoichiometry ratio, the above reaction leads to relative rates of disappearance or generation:

$-r_{\text {EthyAcetate }}=-r_{\mathrm{NaOH}}=r_{\mathrm{C}_{2} \mathrm{H}_{5} \mathrm{OH}}=r_{\mathrm{CH}_{3} \mathrm{CO}_{2} \mathrm{Na}}$

The kinetics of alkaline hydrolysis of ethyl acetate can be represented by a power-law model equation; the specific reaction rate of $\mathrm{NaOH}$ (species A) is as follows:

$-r_{A}=k C_{A}^{\alpha} C_{B}^{\beta}$

where $C_{A}$ is the $\mathrm{NaOH}$ concentration ( $\left.\mathrm{mol} / \mathrm{l}\right), C_{B}$ is the ethyl acetate concentration ( $\mathrm{mol} / \mathrm{l}), \alpha$ and $\beta$ are the reaction order with respect to $\mathrm{NaOH}$ and ethyl acetate respectively; the reaction rate constant $(k)$ depends on the temperature following the Arrhenius form

$k=A * \exp \left(-E_{a} / R T\right)$

where $E_{a}$ is the activation energy $(\mathrm{kJ} / \mathrm{mol}), A$ is the preexponential factor (liter/mol-sec), $\mathrm{R}$ is the ideal gas constant $(\mathrm{J} / \mathrm{mol}-\mathrm{K})$, and $T$ is the absolute temperature $(K)$. The element balance in a continuous stirred-tank reactor (CSTR) was used to investigate the kinetics under transient conditions, which can be expressed as follows:

$\frac{d N_{A}}{d t}=F_{A O}-F_{A}+\int_{V} r_{A} d V$

where $N_{A}$ is number of moles in the reaction ( $\left.\mathrm{mol} / \mathrm{l}\right)$, $F_{A}$ is the molar flow rate of $\mathrm{NaOH}(\mathrm{mol} / \mathrm{s}) r_{A}$ is the rate of reaction (mol/l-s), and $V$ is the vessel size $\left(\mathrm{cm}^{3}\right)$. For the liquid phase, the performance equation for the CSTR reactor can also be written in terms of concentration when there is no volume change, i.e., $F_{A O}=C_{A O} v_{A O}, F_{A}=C_{A} v_{A}$

$\frac{d C_{A}}{d t}=\frac{1}{\tau}\left(C_{A 0}-C_{A}\right)+r_{A}$

where $v_{0}=v_{A 0}+v_{B 0}$ and $\tau=V_{R} /\left(v_{A 0}+v_{B 0}\right)$ can also be written in the mass balance equation of the system: Ethyl acetate:

$\frac{d C_{B}}{d t}=\frac{1}{\tau}\left(C_{B 0}-C_{B}\right)+r_{B}=\frac{1}{\tau}\left(C_{B 0}-C_{B}\right)+r_{A}$

Ethanol:

$\frac{d C_{C}}{d t}=\frac{1}{\tau}\left(0-C_{C}\right)+r_{C}=r_{A}-\frac{1}{\tau} C_{C}$

Sodium acetate:

$\frac{d C_{D}}{d t}=r_{D}-\frac{1}{\tau} C_{D}=r_{A}-\frac{1}{\tau} C_{D}$

For our transient kinetic study, a continuous change of $\mathrm{NaOH}$ concentration of the inlet stream to the reactor was generated by injecting a controlled dose of $\mathrm{NaOH}$ into the mixing tank. This technique 
is called "composition modulation (CM) technique", in which the molar flow rate of $\mathrm{NaOH}$ solution outlet from mixing can be expressed as follows:

$\delta_{A(t)}=C_{A m i} v_{m}+\left[C_{A}^{\delta}\left(V^{\delta} / t^{\delta}\right)\right]$

Equation 10 can also be written in terms of concentration:

$C_{A m 0}(t)=\frac{\delta_{A}}{\left(v_{m}+\left(V^{\delta} / t^{\delta}\right)\right)}$

Combining TS and CM enables us to obtain a sufficient amount of kinetic data for estimating kinetic parameters in a very short period of time compared to the conventional kinetic study. According to our technique, variations in reaction temperature and $\mathrm{NaOH}$ concentration in the CSTR reactor can be collected during the experiment. After that, this information will be translated into the set of kinetic data such as the order of reaction; the activation energy and the pre-exponential factor. Combinations of parameters in the specified ranges are generated in order to simulate the output profile of $\mathrm{NaOH}$ from the reactor. This pattern will be compared with the actual $\mathrm{NaOH}$ concentration collected from the experiment. The combination of parameters that provides the minimum sum of squared errors will be used to represent the kinetic data of this reaction (see [8] for details).The sum of squared error is defined by:

$S S E=\sum\left(C_{A e x}-C_{A c a l}\right)^{2}$

Where $C_{A e x}$ is the $\mathrm{NaOH}$ concentration from the experiment and $C_{\text {Acal }}$ is the $\mathrm{NaOH}$ concentration from the mathematical model.

\subsection{Sampling frequency}

Noise constitutes an unwanted signal which contaminates the output signal. It causes the data to be incorrect with respect to the actual data. Noise may originate from a measurement device, electrical equipment or the process itself. This research concerns about constraints over noise for the accuracy of kinetic information extracted via TS-CM CSTR technique. The suitable sampling frequency range will be considered for this transient kinetic study in order to obtain raw data that provides the least error to the kinetic parameters (compared to those obtained from the $\mathrm{NaOH}$ concentration profile that is undistorted by noise). In this work, unwanted signal intensity represented by random noise was added to the output signal of $\mathrm{NaOH}$ concentration from CSTR $(0.1,0.3,0.5,1.0,3.0$, and $5.0 \%)$. The noise intensity was calculated as

Noise $(\%)=100 \times\left(\frac{y-\hat{y}}{y}\right)$

where $y$ is the data contaminated with noise signal and $\hat{y}$ is the data without noise signal.

\subsection{Digital filter}

To reduce the impact of noise signal on the accuracy of our extracted kinetic parameters, a smoothing filter was applied on the profile of $\mathrm{NaOH}$ concentration at the exit of the CSTR. A good filter lets the relevant information pass through while blocking the noise signal. Due to simplicity of operating equation, digital filtering is used for estimating kinetic parameters. For this work, three important types of low-pass digital filters are considered: the exponential filter or first-order filter, the double exponential filter or second-order filter, and the Savitzky-Golay filter.

\subsection{Exponential filter or first-order filter}

The exponential or first-order filter is the low-pass filter commonly used in most processes [10]. Its operation is described by a first order differential equation and can be expressed as

$\tau_{\mathrm{F}} \frac{\mathrm{d} Y(t)}{\mathrm{d} t}+Y(t)=X(t)$

Rearranging gives

$Y_{\mathrm{j}}=\frac{\Delta t}{\tau_{\mathrm{F}}+\Delta t} X_{\mathrm{j}}+\frac{\tau_{\mathrm{F}}}{\tau_{\mathrm{F}}+\Delta t} Y_{\mathrm{j}-1}$

We define the dimensionless quantity $\alpha=\frac{\Delta t}{\tau_{F}+\Delta t}$;
where $0<\alpha \leq 1$

As a result, equation (15) can be written as

$Y_{j}=\alpha X_{j}+(1-\alpha) Y_{j-1}$ 
Where $X_{j}$ is measured data, $Y_{j}$ is filtered value and $\tau_{F}$ is the filter time constant.

Note that when $\alpha \rightarrow 1$, the filtering is suppressed (the filter output is the measured data $\left(X_{j}\right)$ ). On the other hand, when $\alpha \rightarrow 0$, the current measured data tends to be ignored. Therefore, the choice of filter parameter must be carefully considered for smoothing data.

\subsection{Double exponential filter or second-order filter}

Another useful digital filter is the double exponential or second-order filter, which offers some advantages compared with the exponential filter-namely it provides better filtering of high-frequency noise. The operating equation of a double exponential filter can be expressed as:

$\bar{Y}_{j}=\alpha^{2} X_{j}+2(1-\alpha) \bar{Y}_{j-1}-(1-\alpha)^{2} \bar{Y}_{j-2}$

Similar to the exponential filter, the filter parameter was considered as well. The double exponential filter performance is superior for blocking the relevant noise compared to the exponential filter. However, the latter is still applied to more applications due to the fact that the double exponential filter is more complicated than the exponential filter.

\subsection{Savitzky-Golay filter}

The Savitzky-Golay filter or least-squares smoothing filter is the simplest type of digital filter that has been used in a wide range of applications such as spectroscopy in chemical engineering field [13], chromatograms and IR spectra [14] because this method can eliminate noise while significant characteristics of the data such as peak height and width are preserved [15]. This filter calculates a smoothing value based on the least-squares regression, which can be affected by the polynomial degree and sliding length [16]. Therefore, the combination of both will be considered for the smoothing data from the output $\mathrm{NaOH}$ concentration in this work.

The Savitzky-Golay filter is defined as follows:

$g_{i}=\sum_{n=-n_{L}}^{n_{R}} c_{n} f_{i+n}$

Where $f_{i+n}$ is the original data, $g_{i}$ is the output data,
$n_{L}$ denotes the number of points to the left of data, $n_{R}$ denotes the number of points to the right of data, and $C_{n}$ is a Savitztky-Golay coefficient which can be calculated by

$c_{n}=\left\{\left((A)^{T} A\right)^{-1}\left((A)^{T} e_{n}\right)\right\}$

Savitzky-Golay is a least square regression therefore $e_{n}$ in equation (19) is replaced by a unit vector and the matrix A is represented by

$A_{i j}=i^{j} \quad i=-n_{L, \ldots,} n_{R} \quad j=0, \ldots, M$

where $M$ is the degree of polynomial.

\section{Results and Discussion}

The first part of this section deals with the signal pattern from the TS-CM CSTR for the alkaline hydrolysis of ethyl acetate. After that, based on our output signal and experimental conditions, the effects of sampling frequency and noise intensity on the accuracy of kinetic parameters are discussed. The last part is the implementation of several methods for noise reduction in order to improve the precision of our extracted reaction kinetics.

\subsection{Response signal from TS-CM CSTR}

The experimental data collected from TS-CM CSTR was used to calculate the $\mathrm{NaOH}$ concentration as shown in Figure 2 for the time-evolution of $\mathrm{NaOH}$ concentration at the exit of the reactor (see [10] for detailed explanation). At the beginning, the reactor system was at a steady state at $31.2^{\circ} \mathrm{C}$. After the pulse injection of $0.8 \mathrm{M} \mathrm{NaOH}$ solution for the period of 3 seconds, $\mathrm{NaOH}$ concentration in the reactor sharply evolved to a maximum followed by an exponential decline as the system underwent relaxation to establish a new steady state. During this process, the temperature of our reacting mixture was ramped from $31.2^{\circ} \mathrm{C}$ to $45^{\circ} \mathrm{C}$. This temperature difference of $14^{\circ} \mathrm{C}$ caused the $\mathrm{NaOH}$ concentration to be lower than the initial value due to the increased reactant consumption rate.

The transient responses of reactant concentration and reaction temperature are directly related to the reaction kinetics. By systematically varying the kinetic parameters of this reaction, the mathematical 


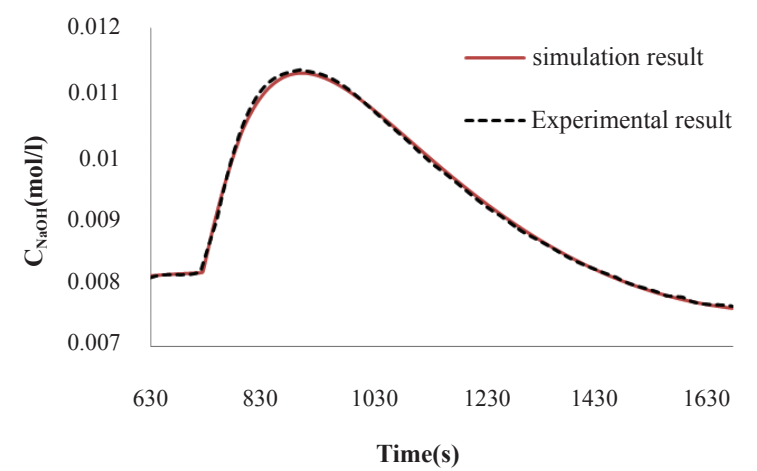

Figure 2: The concentration profile of $\mathrm{NaOH}$ : comparison between the simulated results and the experimental results.

model developed previously was used to search for the set of parameters providing the closest match with $\mathrm{NaOH}$ concentration profile obtained experimentally (minimum sum of squared errors). The kinetic parameters were: $\alpha=1, \beta=1, E_{a}=48.6 \mathrm{~kJ} / \mathrm{mol}$, and $A r=3.95 \times 10^{7} 1 / \mathrm{mol}-\mathrm{sec}$.

\subsection{Sampling frequency and noise intensity}

According to the obtained kinetic parameters, the continuous baseline signal of $\mathrm{NaOH}$ concentration was generated in order to study the effect of sampling frequency and noise intensity on the accuracy of kinetic parameters. This baseline signal was subject to modification in terms of data points and degree of scattering resulting in distorted signal. For the first part, different degrees of noise intensity $(0.5,1.0$, 3.0 , and $5.0 \%$ ) were added to the output signal of $\mathrm{NaOH}$ concentration while the sampling period was kept constant at 1 second. Figure 3 shows the effect of noise on the concentration profile; the black dotted line represents the actual $\mathrm{NaOH}$ concentration and the red line represents the $\mathrm{NaOH}$ concentration which was contaminated by noise. Apparently, the $\mathrm{NaOH}$ concentration signal which was contaminated by noise increasingly deviates from the theoretical data with increased degree of noise intensity.

Based on the sum of squared errors, each distorted signal was processed through a mathematical model for the selection of kinetic parameters of the alkaline hydrolysis of ethyl-acetate. The obtained kinetic parameters are listed in Table1.

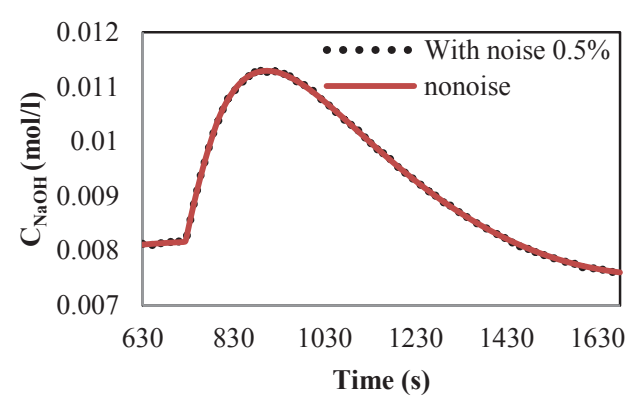

(a)

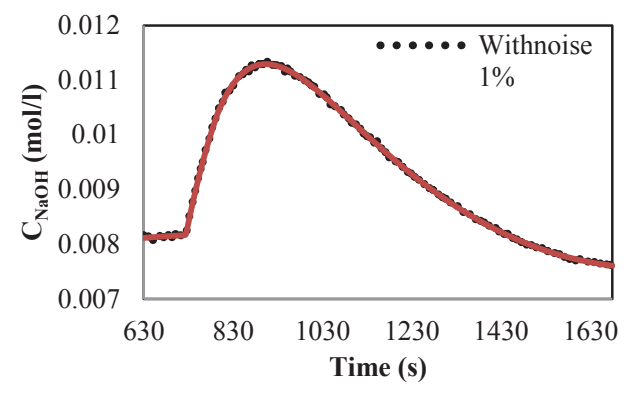

(b)

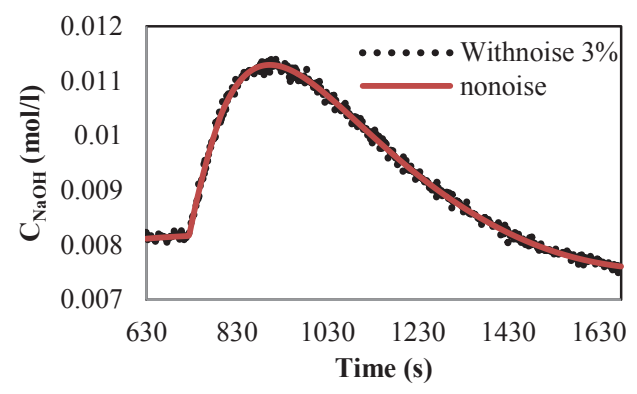

(c)

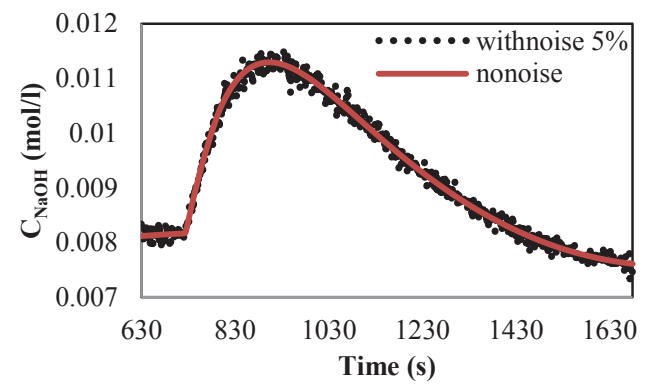

(d)

Figure 3: The concentration profile of $\mathrm{NaOH}$ contaminated with various degrees of noise intensity: (a) $0.5 \%$, (b) $1 \%$, (c) $3 \%$, and (d) $5 \%$. 
Table 1: Influence of noise intensity on the kinetic parameters of alkaline hydrolysis ethyl acetate by TS-CM CSTR technique

\begin{tabular}{|c|c|c|c|c|}
\hline \multirow{2}{*}{ Noise intensity } & \multicolumn{4}{|c|}{ Kinetic parameter } \\
\cline { 2 - 5 } & $\boldsymbol{\alpha}$ & $\boldsymbol{\beta}$ & $\begin{array}{c}\boldsymbol{E}_{\boldsymbol{a}} \\
(\mathrm{kJ} / \mathrm{mol})\end{array}$ & $\begin{array}{c}\boldsymbol{A r} \\
\text { (liter/mol-sec) }\end{array}$ \\
\hline- & 1 & 1 & 48.60 & $3.95 \times 10^{7}$ \\
\hline 0.5 & 1 & 1 & 48.56 & $3.89 \times 10^{7}$ \\
\hline 1 & 1 & 1 & 48.34 & $3.58 \times 10^{7}$ \\
\hline 3 & 1.2 & 0.8 & 48.18 & $3.48 \times 10^{7}$ \\
\hline 5 & 1.2 & 0.8 & 48.06 & $3.32 \times 10^{7}$ \\
\hline
\end{tabular}

For small degree of noise intensity $(0.5-1 \%)$, the reaction orders with respect to $\mathrm{NaOH}$ and ethylacetate are still the same as those obtained from the process without noise added-that is, $\alpha=1, \beta=1$ but the activation energy and pre-exponential factor are slightly changed. Further increasing the noise intensity to 3 or $5 \%$ leads to significant deviation of the kinetic parameters: see Table 1. In fact, deviations in the kinetic parameters increase with increased noise intensity. Thus, in estimating the kinetic parameters by TS-CM CSTR, the intensity of noise must always be a concern.

This part, the effect of sampling interval on the accuracy of kinetic parameters via TS-CM CSTR technique is considered. The baseline signal was discretized in order to simulate the data collection with sampling interval of 1,30 , and 60 seconds. Again, different noise intensities $(0.5,1.0,3.0$, and $5.0 \%$ ) were added to the signal. These sets of modified signal were then used to determine the kinetic parameters. Results indicate that the deviation of the kinetic parameters increased with increasing time interval as shown in Table 2. This is because a lower sampling frequency causes the loss of some important data points and ultimately results in a lowered accuracy. The combined effect of noise intensity and long sampling period noticeably worsen the estimation of kinetic parameters i.e. 1 second with $1 \%$ noise vs 60 seconds with $1 \%$ noise. It appears that our system can be used for $1 \%$ noise with the sampling interval as long as 30 seconds with a slight deviation in kinetic parameters.
Table 2: Influence of sampling frequency on the kinetic parameters of alkaline hydrolysis of ethyl acetate by TS-CM CSTR technique

\begin{tabular}{|c|c|c|c|c|c|}
\hline \multirow{2}{*}{$\begin{array}{c}\text { Noise } \\
\text { intensity } \\
(\mathbf{\%})\end{array}$} & \multirow{2}{*}{$\begin{array}{c}\text { Sampling } \\
\text { time(s) }\end{array}$} & \multicolumn{4}{|c|}{ Kinetic parameters } \\
\cline { 3 - 6 } & - & $\boldsymbol{\beta}$ & $\begin{array}{c}\boldsymbol{E}_{\boldsymbol{a}} \\
(\mathbf{k J} / \mathbf{m o l})\end{array}$ & $\begin{array}{c}\boldsymbol{A} \boldsymbol{r} \\
\text { (liter/mol-sec) }\end{array}$ \\
\hline \multirow{3}{*}{0.5} & - & 1 & 1 & 48.60 & $3.95 \times 10^{7}$ \\
\cline { 2 - 6 } & 30 & 1 & 1 & 48.56 & $3.89 \times 10^{7}$ \\
\cline { 2 - 6 } & 60 & 1 & 1 & 48.32 & $3.55 \times 10^{7}$ \\
\hline \multirow{4}{*}{1} & 1 & 1 & 1 & 48.34 & $3.52 \times 10^{7}$ \\
\cline { 2 - 6 } & 30 & 1 & 1 & 48.46 & $3.75 \times 10^{7}$ \\
\cline { 2 - 6 } & 60 & 1.6 & 0.4 & 47.94 & $3.39 \times 10^{7}$ \\
\hline \multirow{3}{*}{3} & 1 & 1.2 & 0.8 & 48.18 & $3.48 \times 10^{7}$ \\
\cline { 2 - 6 } & 30 & 1.4 & 0.6 & 48.18 & $3.60 \times 10^{7}$ \\
\cline { 2 - 6 } & 60 & 1.4 & 0.56 & 47.98 & $3.22 \times 10^{7}$ \\
\hline \multirow{3}{*}{5} & 1 & 1.2 & 0.8 & 48.06 & $3.32 \times 10^{7}$ \\
\cline { 2 - 6 } & 30 & 1.32 & 0.64 & 48.34 & $3.32 \times 10^{7}$ \\
\cline { 2 - 6 } & 60 & 1.64 & 0.28 & 47.86 & $3.05 \times 10^{7}$ \\
\hline
\end{tabular}

\subsection{Noise reduction}

It is evident from the previous section that the noise intensity of $3 \%$ and $5 \%$ drastically reduces the accuracy of kinetic parameters obtained by TS-CM CSTR technique. In this section, we explore the possibility to improve the accuracy by noise reduction method. The distorted signal with 5\% noise and the sampling period of 1 second is used to test the performance of three digital filters (exponential filter, double exponential filter, and Savitzky-Golay filter) for their ability to eliminate the effects of noise on kinetic parameters. The influences of the filter parameter $(0.1,0.3,0.5$, and 0.7$)$ were studied for both exponential and double exponential filters. Figure 4 shows the influence of a on the sum of squares of error. From this comparison of ability for attenuation of noise intensity, it appears that the double exponential filter provides better filtering than the exponential filter; this can simply be seen from the lower sums of the squares of error. In addition, when we consider the influence of the filter parameter $(\alpha)$, we can see that the attenuation of noise is high when a is low for both the exponential and double exponential filters. Excepting the case of $\alpha=0.1$, which shows the opposite trend, it can be seen that a higher sum of the squared errors (SSE) is obtained when using double exponential filter as compared to the other filter type. This high SSE indicates that greater deviation from 


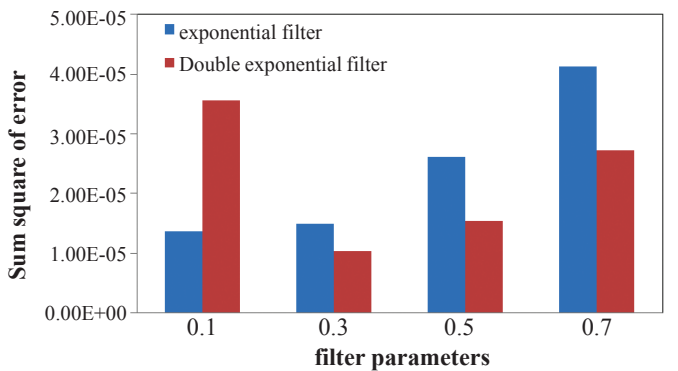

Figure 4: Comparison of the sum of the squared errors of the exponential filter and the double exponential filter for $5 \%$ noise.

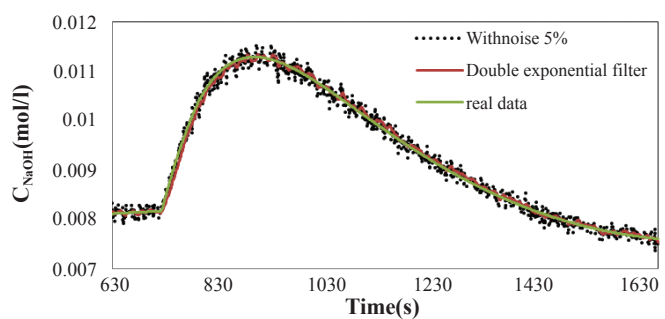

Figure 5: Output using a double exponential $\alpha=0.3$.

the real data (due to less elimination of noise or due to smoothing of the data) causes an increase in the amount of time-lag in the obtained data. Therefore, the balance between the degree of smoothness and the lag-time must be optimized. The lowest SSE occurs when the double exponential filter $\alpha=0.3$ was applied for eliminating noise in the data. Figure 5 shows the output data obtained by using a double exponential filter $\alpha=0.3$, which successfully removes noise without much deviation from the real data.

After eliminating noise via a double exponential filter, the output data was used to estimate kinetic parameters via the dynamic simulation of the mathematical model as mentioned above (see section 2). Results are summarized in Table 3. Although the double exponential filter can eliminate noise from the signal better than the exponential filter, when we consider the kinetic parameters (e.g. reaction order, activation energy and pre-exponential factor), it can still be found that the kinetic parameters deviate from the obtained kinetic parameters in without-noise case. A double exponential filter is unable to smooth data while preserving the data features and, for this reason, we obtain inaccuracy in the kinetic parameters.

Since both, the double exponential filter and exponential filter yielded kinetic parameters that
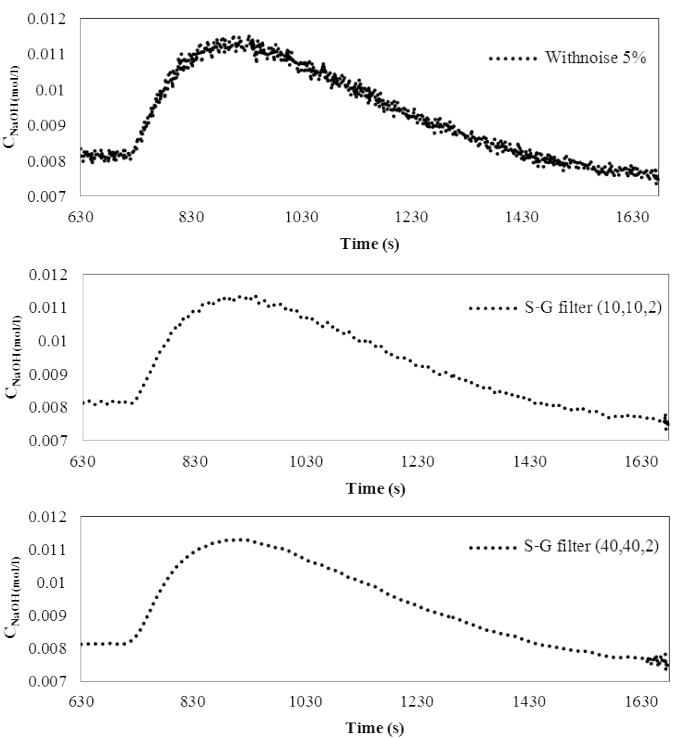

Figure 6: Results of window size influence on the ability to eliminate noise by the Savitzky-Golay filter. Top: concentration profile of $\mathrm{NaOH}$ contaminated with $5 \%$ noise. Center: result of smoothing data by Savitzky-Golay filter (window size: 21 points). Bottom: result of data smoothing by Savitzky-Golay filter (window size: 81 points).

significantly deviated from the true values, we then applied the Savitzky-Golay (S-G filter) for eliminating noise from the data for $\mathrm{NaOH}$ concentration. The performance of the Savitzky-Golay was tested by considering the optimal parameters for noise elimination and data smoothing by varying the window size (number of data points, $2 \mathrm{~N}+1$ ) and polynomial degree (M). Figure 6 illustrates the effect of different window size on the noise intensity; the results indicate that the signal data is more smoothed with a larger window size, polynomial degree. It appears that a window size of 81 points leads to superior data smoothing and upon visual inspection reduces noise amplitude compared to that of a window size of 21 points.

Table 3: Influence of filter parameters on the obtained kinetic parameters

\begin{tabular}{|c|c|c|c|c|c|}
\hline \multirow{2}{*}{$\begin{array}{c}\text { Noise } \\
\text { intensity (\%) }\end{array}$} & \multirow{2}{*}{$\begin{array}{c}\text { Filter } \\
\text { parameter }\end{array}$} & \multicolumn{4}{|c|}{ Kinetic parameters } \\
\cline { 3 - 6 } & & $\boldsymbol{\beta}$ & $\begin{array}{c}\boldsymbol{E}_{\boldsymbol{a}} \\
(\mathbf{k J} / \mathbf{m o l})\end{array}$ & $\begin{array}{c}\boldsymbol{A r} \\
\text { (liter/mol-sec) }\end{array}$ \\
\hline- & - & 1.00 & 1.00 & 48.60 & $3.95 \times 10^{7}$ \\
\hline 5 & - & 1.20 & 0.80 & 48.06 & $3.32 \times 10^{7}$ \\
\hline 5 & 0.3 & 1.76 & 0.44 & 47.04 & $2.66 \times 10^{7}$ \\
\hline
\end{tabular}


The smoothed data obtained from the SavitzkyGolay filter was used to extract the kinetic parameters. Results are summarized in Table 4. The simulation results indicate that window sizes of 21 and 81 points successfully improved the accuracy of the kinetic parameters compared to those obtained from the double exponential filter. The results for the case with a window size of 21 points significantly improved the accuracy in terms of reaction orders compared to the case without noise reduction. Note that the deviation from the true kinetics can be further reduced by increasing the window size to 81 . We also investigated the effect of polynomial order for Savitzky-Golay filter on the noise reduction performance and found that the sum of squared errors could be decreased slightly by increasing the polynomial order from 2 to 4 . Nevertheless, the accuracy of kinetic parameters did not significantly change.

Table 4: Influence of frame size on the obtained kinetic parameters

\begin{tabular}{|c|c|c|c|c|c|}
\hline \multirow[b]{2}{*}{$\begin{array}{c}\text { Noise } \\
\text { intensity } \\
(\%)\end{array}$} & \multirow[b]{2}{*}{$\begin{array}{c}\text { Window } \\
\text { size }\end{array}$} & \multicolumn{4}{|c|}{ Kinetic parameters } \\
\hline & & $\alpha$ & $\beta$ & $\begin{array}{c}E_{a} \\
(\mathrm{~kJ} / \mathrm{mol})\end{array}$ & $\begin{array}{c}A r \\
\text { (liter/ } \\
\text { mol-sec) }\end{array}$ \\
\hline- & - & 1.00 & 1.00 & 48.60 & $3.95 \times 10^{7}$ \\
\hline 5 & - & 1.20 & 0.80 & 48.06 & $3.32 \times 10^{7}$ \\
\hline 5 & 10 & 1.00 & 1.00 & 48.08 & $3.24 \times 10^{7}$ \\
\hline 5 & 40 & 1.00 & 1.00 & 48.16 & $3.35 \times 10^{7}$ \\
\hline
\end{tabular}

\section{Conclusions}

For a faster method of estimating kinetic parameters compared to the conventional method known as TS-CM technique, the quality of signal collected from transient experimentation is of major importance for the accuracy of parameters obtained. Increasing the noise intensity and/or lowering the sampling frequency increases the deviation of signal resulting in poor estimates of kinetic parameters. Different types of filter including exponential filter, double exponential filter, and SavitzkyGolay filter were employed in order to improve the accuracy of estimated parameters. The latter exhibits superior performance for smoothing data especially with large window size. Hence, the combination of TS-CM technique and the use of SavitzkyGolay filter can help decrease the time, resource, and effort for achieving information on reaction kinetics without compromising the accuracy.

\section{References}

[1] O. Levenspiel, Chemical reaction engineering, 3rd ed. United States of America: John Wiley \& Sons, 1999.

[2] Y. Tanawannapong, A. Kaewchada, and A. Jaree, "Biodiesel production from waste cooking oil in a microtube reactor," J. Ind. Eng. Chem., vol. 19, pp. 37-41, Jan. 2013.

[3] Y. Khan, M. Marin, R. Karinen, J. Lehtonen, and J. Kanervo, "1-Butanol dehydration in microchannel reactor: Kinetics and reactor modelling," Chem. Eng. Sci., vol. 137, pp. 740751, Dec. 2015.

[4] L. Zhang and Z. Haidong, "Reaction mechanism and forced concentration oscillation characteristics of benzene oxidation process," Chinese J. Chem. Eng., vol. 1, pp. 30-37, Jan. 1993.

[5] S. P. Asprey, B. W. Wojciechowski, N. M. Rice, and A. Dorcas, "Application of temperature scanning in kinetic investigations: The hydrolysis of acetic anhydride," Chem. Eng. Sci., vol. 51, pp. 4681-4692, Oct. 1996.

[6] S. B. Domke, R. F. Pogue, F. J. R. Van Neer, and C. M. Smith, "Investigation of the kinetics of ethylbenzene pyrolysis using a temperaturescanning reactor," Ind. Eng. Chem. Res., vol. 40, pp. 5878-5884, Dec. 2001.

[7] B. W. Wojciechowski, S. P. Asprey, N. M. Rice, and A. Dorcas, "Kinetic studies using temperaturescanning: the oxidation of carbon monoxide," Appl. Catal. A-Gen., vol. 190, pp. 1-24, Jan. 2000.

[8] B. Suman, "Study of unsteady state process operation," Chem. Eng. J., vol. 104, pp. 63-71, Nov. 2004.

[9] R. Lange, R. Gutsche, and J. Hanika, "Forced periodic operation of a trickle-bed reactor," Chem. Eng. Sci., vol. 54, pp. 2569-2573, Jul. 1999.

[10] A. Jaree and C. Naammaneerat, "A kinetics study in CSTR using simultaneous temperature scanning and composition modulation: The alkaline hydrolysis of ethyl acetate," Can. J. Chem. Eng., vol. 88, pp. 1027-1033, Dec. 2010.

[11] P. Phanawadee and B. Wisuthchai, "Characteristic of surface concentration distribution due to irreversible adsorption in a one-pulse TAP 
experiment," Chem. Eng. Sci., vol. 65, pp. 2525-2429, Apr. 2010.

[12] R. M. Oisiovici, S. L. Cruz, and J. A. F. R. Pereira, "Digital filtering in the control of a batch distillation column," ISA T., vol. 38, pp. 217-224, Jul. 1999.

[13] D. J. W. Ng and M. Assirelli, "Mixing study in batch stirred vessels using a fibre-obtic uv-vis monitoring technique a novel method," Chem. Eng. Res. Des., vol. 85, pp. 1348-1354, Oct. 2007.

[14] P. Barak, "Smoothing and Differentiation by an adaptive-degree polynomial filter," Anal. Chem., vol. 67, pp. 2758-2762, Sep. 1995.

[15] J. L. Guinon, E. Ortega, J. G. Anton, and V. P. Herranz, "Moving average and Savitzki-Golay smoothing filters using matcad," presented at Int. Conf. on Engineering Education, Coimbra, Portugal, Sep. 3-7, 2007.

[16] W. H. Press, S. A. Teukdsky, W. T. Vetterling, and B. P. Flannery, Numerical recipes in Fortran 77: The art of scientific computing, 2 nd ed. New York: Press Syndicate of the University of Cambridge, 1992. 